\title{
Solution of Axisymmetric Potential Problem in Oblate Spheroid Using the Exodus Method
}

\author{
O. D. Momoh, ${ }^{1}$ M. N. O. Sadiku, ${ }^{2}$ and S. M. Musa ${ }^{2}$ \\ ${ }^{1}$ College of Engineering, Technology, and Computer Science, Indiana University-Purdue University, Fort Wayne, IN 46805, USA \\ ${ }^{2}$ Roy G. Perry College of Engineering, Prairie View A\&M University, Prairie View, TX 77446, USA
}

Correspondence should be addressed to O. D. Momoh; momohd@ipfw.edu

Received 7 November 2013; Accepted 15 February 2014; Published 17 March 2014

Academic Editor: Quan Yuan

Copyright (C) 2014 O. D. Momoh et al. This is an open access article distributed under the Creative Commons Attribution License, which permits unrestricted use, distribution, and reproduction in any medium, provided the original work is properly cited.

\begin{abstract}
This paper presents the use of Exodus method for computing potential distribution within a conducting oblate spheroidal system. An explicit finite difference method for solving Laplace's equation in oblate spheroidal coordinate systems for an axially symmetric geometry was developed. This was used to determine the transition probabilities for the Exodus method. A strategy was developed to overcome the singularity problems encountered in the oblate spheroid pole regions. The potential computation results obtained correlate with those obtained by exact solution and explicit finite difference methods.
\end{abstract}

\section{Introduction}

An oblate spheroid is the surface generated by the rotation of an ellipse about its minor axis, and depending upon the ellipse's eccentricity, the spheroid will be flattened about the minor axis [1]. An oblate spheroidal shell, for instance, is considered as a continuous system constructed from two spherical shell caps by matching the continuous boundary conditions [2].

Oblate and prolate spheroidal coordinates are widely used in many fields of science and engineering, such as potential theory, fluid mechanics, heat and mass transfer, thermal stress, and elastic inclusions. For example, oblate and prolate spheroids being surfaces of revolution can be more easily conformed to most districts of human body (e.g., extremities) which is of interest for dedicated MRI systems [3]. Oblate spheroidal coordinates are the natural choice for the translation of any ellipsoid parallel to a principal axis [4]. There is a more recent improvement in the lightning ground tracking systems based on the time-of-arrival (TOA) technique because of the refinement in the mathematics to more accurately accommodate the oblate shape of the earth spheroid. Approximating the earth as a perfect sphere affects not only the accuracy of time clock offset calculations, but also the accuracy of stroke coordinate computation given receiver time differences. Oblate solution mathematics can provide a substantial systematic error reduction of up to $50 \%$ percent [5].

In this paper, Exodus method is used to compute potential distribution inside conducting oblate spheroidal shells maintained at two potentials. This work is a continuation of our previous work in which a fixed random walk Monte Carlo method (MCM) was used for numerical computation of potential distribution with two conducting oblate spheroidal shells maintained at two potentials [6]. An explicit Neumann boundary condition was imposed at the pole regions $(\eta=$ $-\pi / 2, \pi / 2)$ of the oblate spheroid to treat the presence of singularities in those regions.

The Exodus method is one of Monte Carlo methods which are nondeterministic (probabilistic or stochastic) numerical methods employed in solving mathematical and physical problems. The fixed random walk and Exodus methods are the most frequently used Monte Carlo methods for solving heat conduction and potential problems. The Exodus method is however preferred to fixed random walk method because of its computational efficiency. It yields more 


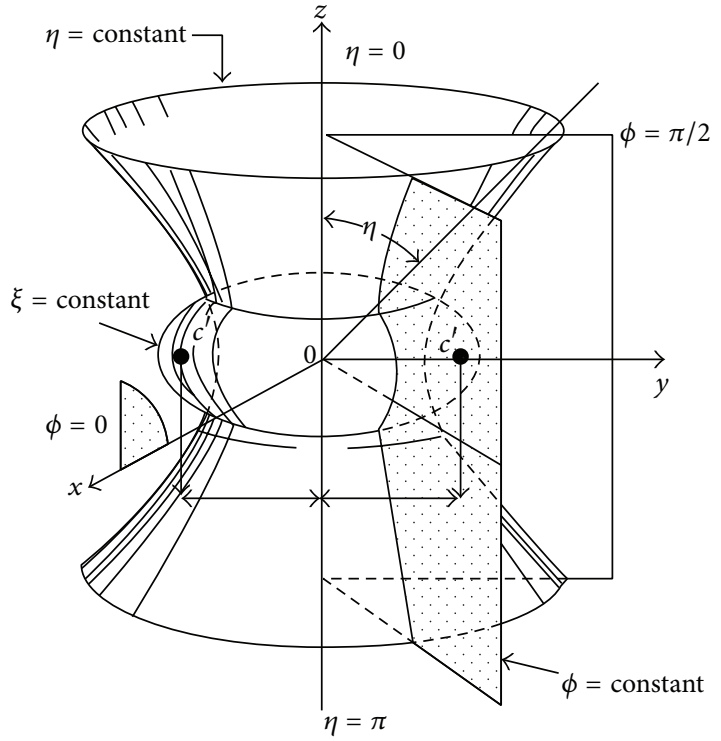

Figure 1: The oblate spheroidal coordinate system [9].

accurate results with less computing time as compared to the original Monte Carlo method [7, 8].

\section{Oblate Spheroidal Coordinate Systems}

The geometry of an oblate spheroid showing surfaces of constant oblate spheroidal coordinates is illustrated in Figure 1. The oblate spheroidal coordinates are related to the rectangular coordinates as follows:

$$
\begin{aligned}
& x=c^{\prime} \cosh \xi \sin \eta \cos \varphi, \\
& y=c^{\prime} \cosh \xi \sin \eta \sin \varphi, \\
& x=c^{\prime} \sinh \xi \cos \eta,
\end{aligned}
$$

where $c^{\prime}$ is the focal length of the oblate spheroid.

An arbitrary grid point $r$ on the oblate spheroid is given by

$$
\bar{r}_{i, j, k}=\xi_{i} \bar{a}_{\xi}+\eta_{j} \bar{a}_{\eta}+\phi_{k} \bar{a}_{\phi}
$$

where $\bar{a}_{\xi}, \bar{a}_{\eta}$, and $\bar{a}_{\phi}$ are the unit vectors in the direction of $\xi$, $\eta$, and $\phi$ coordinates, respectively.

The respective scale factor for each of the three coordinates $(\xi, \eta, \phi)$ is

$$
\begin{aligned}
& h_{\xi}=c^{\prime} \sqrt{\sinh ^{2} \xi+\sin ^{2} \eta}, \\
& h_{\eta}=c^{\prime} \sqrt{\sinh ^{2} \xi+\sin ^{2} \eta}, \\
& h_{\phi}=c^{\prime} \cosh \xi \cos \eta .
\end{aligned}
$$

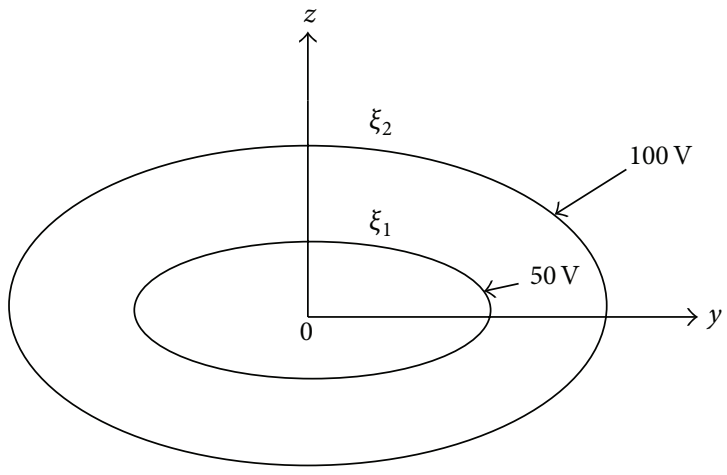

FIGURE 2: Two oblate spheroidal shells.

\section{Potential Distribution Computation}

3.1. Finite Difference Transformation of Oblate Spheroid Laplace's Equation. The Laplacian equation in oblate spheroidal coordinate systems is

$$
\begin{aligned}
0=\nabla^{2} V & =\left(\frac{1}{\sinh ^{2} \xi+\sin ^{2} \eta}\right) \\
* & {\left[\left(\operatorname{sech}^{2} \xi \tan ^{2} \eta+\sec ^{2} \eta \tanh ^{2} \xi\right) \frac{\partial^{2} V}{\partial \phi^{2}}\right.} \\
& \left.+\frac{\partial^{2} V}{\partial \xi^{2}}+\tanh \xi \frac{\partial V}{\partial \xi}+\frac{\partial^{2} V}{\partial \eta^{2}}-\tan \eta \frac{\partial V}{\partial \eta}\right]
\end{aligned}
$$

The term outside the square bracket may be ignored. Also the first term inside square bracket is ignored due to the rotational symmetry about the vertical $(z)$ axis. Therefore, (4) reduces to

$$
\frac{\partial^{2} V}{\partial \xi^{2}}+\tanh \xi \frac{\partial V}{\partial \xi}+\frac{\partial^{2} V}{\partial \eta^{2}}-\tan \eta \frac{\partial V}{\partial \eta}=0
$$

Equation (5) governs potential distribution in an axisymmetric oblate spheroid potential problem.

Two oblate spheroidal shells made up of two constant conducting surfaces $\xi_{1}$ and $\xi_{2}$ are shown in Figure 2. The two equipotential surfaces are maintained at $50 \mathrm{~V}$ and $100 \mathrm{~V}$ (Dirichlet boundary conditions), respectively. The choice of these Dirichlet boundary conditions is arbitrary. Any potential value can be assigned. Also, the value of the constant oblate spheroidal surfaces equipotential that constitutes the two conducting shells are arbitrarily chosen as $\xi_{1}=1.0$ and $\xi_{1}=2.0$, respectively. 
The explicit finite difference transformation of (5) is

$$
\begin{aligned}
& V(i, j) \\
& =0.5\left[\frac{1}{\left[\left(1 /(\Delta \xi)^{2}\right)+\left(1 /(\Delta \eta)^{2}\right)\right]}\right] \\
& *\left[V(i+1, j)\left[\frac{1}{(\Delta \xi)^{2}}+\frac{\tanh (i \Delta \xi)}{2 \Delta \xi}\right]\right. \\
& +V(i-1, j)\left[\frac{1}{(\Delta \xi)^{2}}-\frac{\tanh (i \Delta \xi)}{2 \Delta \xi}\right] \\
& +V(i, j+1)\left[\frac{1}{(\Delta \eta)^{2}}-\frac{\tan \left(\eta_{j}\right)}{2 \Delta \eta}\right] \\
& \left.+V(i, j-1)\left[\frac{1}{(\Delta \eta)^{2}}+\frac{\tan \left(\eta_{j}\right)}{2 \Delta \eta}\right]\right] .
\end{aligned}
$$

Figure 3 shows one-quarter of the constant oblate spheroidal surfaces. The figure exhibits symmetry with respect to the $\eta$ coordinate. Therefore, two lines of symmetries will be encountered in this range of $\eta$. They are $\eta=90^{\circ}$ and $\eta=0^{\circ}$. On these lines of symmetries, the condition $\partial V / \partial \eta=0$ is imposed. This strategy eliminates the singularity causing term $\left(\tan \left(\eta_{j}\right) / 2 \Delta \eta\right)$ at the oblate spheroid poles as seen in (6).

Consequently, the finite difference equations along the two lines of symmetries become as follows.

Along $\eta=90^{\circ}, j=j_{\max }$,

$$
\begin{aligned}
& V\left(i, j_{\max }\right) \\
& =0.5\left[\frac{1}{\left[\left(1 /(\Delta \xi)^{2}\right)+\left(1 /(\Delta \eta)^{2}\right)\right]}\right] \\
& *\left[V\left(i+1, j_{\max }\right)\left[\frac{1}{(\Delta \xi)^{2}}+\frac{\tanh (i \Delta \xi)}{2 \Delta \xi}\right]\right. \\
& +V\left(i-1, j_{\max }\right)\left[\frac{1}{(\Delta \xi)^{2}}-\frac{\tanh (i \Delta \xi)}{2 \Delta \xi}\right] \\
& \left.\quad+2 V\left(i, j_{\max }-1\right)\left[\frac{1}{(\Delta \eta)^{2}}\right]\right] .
\end{aligned}
$$

Along $\eta=0^{\circ}, j=0$,

$$
\begin{aligned}
& V(i, 0) \\
& =0.5\left[\frac{1}{\left[\left(1 /(\Delta \xi)^{2}\right)+\left(1 /(\Delta \eta)^{2}\right)\right]}\right] \\
& \quad *\left[V(i+1,0)\left[\frac{1}{(\Delta \xi)^{2}}+\frac{\tanh (i \Delta \xi)}{2 \Delta \xi}\right]\right.
\end{aligned}
$$

$$
\begin{aligned}
& +V(i-1,0)\left[\frac{1}{(\Delta \xi)^{2}}-\frac{\tanh (i \Delta \xi)}{2 \Delta \xi}\right] \\
& \left.+2 V(i, 1)\left[\frac{1}{(\Delta \eta)^{2}}\right]\right] .
\end{aligned}
$$

3.2. Transition Probabilities Determination. In a more compact form, (6) can be rewritten as

$$
\begin{aligned}
V(i, j)= & P_{\xi_{+}} V(i+1, j)+P_{\xi_{-}} V(i-1, j) \\
& +P_{\eta_{+}} V(i, j+1)+P_{\eta_{-}} V(i, j-1),
\end{aligned}
$$

where

$$
\begin{aligned}
& P_{\xi_{-}}=0.5\left(\frac{\left[\left(1 /(\Delta \xi)^{2}\right)+(\tanh (i \Delta \xi) / 2 \Delta \xi)\right]}{\left[\left(1 /(\Delta \xi)^{2}\right)+\left(1 /(\Delta \eta)^{2}\right)\right]}\right), \\
& P_{\xi_{-}}=0.5\left(\frac{\left[\left(1 /(\Delta \xi)^{2}\right)-(\tanh (i \Delta \xi) / 2 \Delta \xi)\right]}{\left[\left(1 /(\Delta \xi)^{2}\right)+\left(1 /(\Delta \eta)^{2}\right)\right]}\right), \\
& P_{\eta_{-}}=0.5\left(\frac{\left[\left(1 /(\Delta \eta)^{2}\right)+(\tan (j \Delta \eta) / 2 \Delta \eta)\right]}{\left[\left(1 /(\Delta \xi)^{2}\right)+\left(1 /(\Delta \eta)^{2}\right)\right]}\right), \\
& P_{\eta_{-}}=0.5\left(\frac{\left[\left(1 /(\Delta \eta)^{2}\right)-(\tan (j \Delta \eta) / 2 \Delta \eta)\right]}{\left[\left(1 /(\Delta \xi)^{2}\right)+\left(1 /(\Delta \eta)^{2}\right)\right]}\right) .
\end{aligned}
$$

Note that $P_{\xi_{+}}+P_{\xi_{-}}+P_{\eta_{+}}+P_{\eta_{-}}=1$. Equations (10a), (10b), (10c), and (10d) serve as the transition probabilities for the Exodus method used in this work. If $N$ particles are dispersed at node $\left(\xi_{0}, \eta_{0}\right)$, they have probabilities $P_{\xi+}, P_{\xi-}, P_{\eta_{+}}$, and $P_{\eta-}$ of moving to points $(\xi+\Delta \xi, \eta),(\xi-\Delta \xi, \eta),(\xi, \eta+\Delta \eta)$, and $(\xi, \eta-\Delta \eta)$, respectively. The direction of movement of the particles is determined by generating a random number $U, 0<U<1$. The particles are instructed to walk as follows:

$$
\begin{array}{ll}
\left(\xi_{0}, \eta_{0}\right) \Longrightarrow\left(\xi_{0}+\Delta \xi, \eta_{0}\right), & \text { if }\left(0<U<P_{\xi_{+}}\right), \\
\left(\xi_{0}, \eta_{0}\right) \Longrightarrow\left(\xi_{0}-\Delta \xi, \eta_{0}\right), & \text { if }\left(P_{\xi_{+}}<U<P_{T}\right), \\
\left(\xi_{0}, \eta_{0}\right) \Longrightarrow\left(\xi_{0}, \eta_{0}+\Delta \eta\right), & \text { if }\left(P_{T}<U<P_{T T}\right), \\
\left(\xi_{0}, \eta_{0}\right) \Longrightarrow\left(\xi_{0}, \eta_{0}-\Delta \eta\right), & \text { if }\left(P_{T T}<U<1\right),
\end{array}
$$

where

$$
P_{T}=P_{\xi_{+}}+P_{\xi_{-}}, \quad P_{T T}=P_{\xi_{+}}+P_{\xi_{-}}+P_{\eta_{+}} .
$$

For the random walk computation along the line of symmetry $\left(\eta=90^{\circ}\right)$, (7) becomes

$$
\begin{aligned}
V\left(i, j_{\max }\right)= & P_{\xi_{+}} V\left(i+1, j_{\max }\right)+P_{\xi-} V\left(i-1, j_{\max }\right) \\
& +P_{\eta_{-}} V\left(i, j_{\max }-1\right)
\end{aligned}
$$




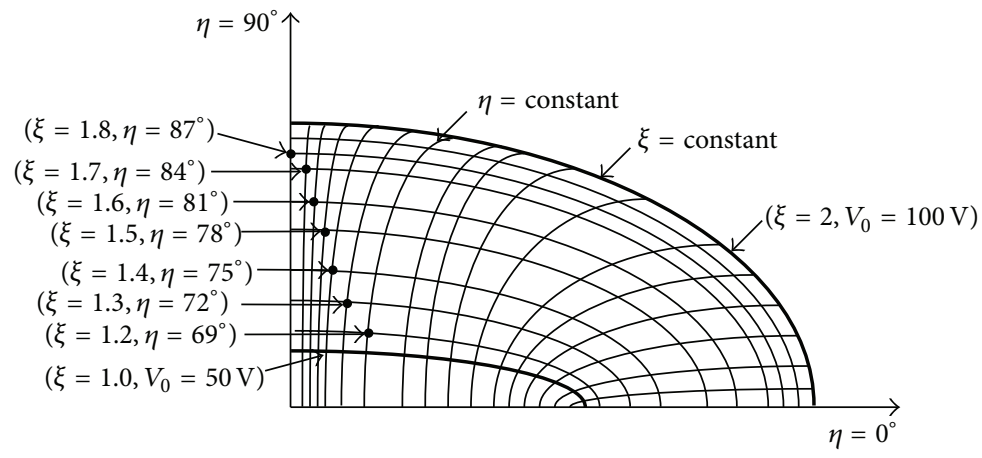

FIGURE 3: Oblate spheroidal surface path used in computing the charge enclosed.

where

$$
\begin{gathered}
P_{\xi-}=0.5\left(\frac{\left[\left(1 /(\Delta \xi)^{2}\right)+(\tanh (i \Delta \xi) / 2 \Delta \xi)\right]}{\left[\left(1 /(\Delta \xi)^{2}\right)+\left(1 /(\Delta \eta)^{2}\right)\right]}\right), \\
P_{\xi_{-}}=0.5\left(\frac{\left[\left(1 /(\Delta \xi)^{2}\right)-(\tanh (i \Delta \xi) / 2 \Delta \xi)\right]}{\left[\left(1 /(\Delta \xi)^{2}\right)+\left(1 /(\Delta \eta)^{2}\right)\right]}\right), \\
P_{\eta^{-}}=\left(\frac{\left[\left(1 /(\Delta \eta)^{2}\right)\right]}{\left[\left(1 /(\Delta \xi)^{2}\right)+\left(1 /(\Delta \eta)^{2}\right)\right]}\right) .
\end{gathered}
$$

Note that $P_{\xi_{+}}+P_{\xi_{-}}+P_{\eta_{-}}=1$ along this line of symmetry. If a random-walking particle is instantaneously at the point $\left(\xi_{0}, 90^{\circ}\right)$, it has probabilities $P_{\xi_{+}}, P_{\xi-}$, and $P_{\eta_{-}}$of moving to points $\left(\xi+\Delta \xi, 90^{\circ}\right),\left(\xi-\Delta \xi, 90^{\circ}\right)$, and $\left(\xi, 90^{\circ}-\Delta \eta\right)$, respectively. The particles are instructed to walk as follows:

$$
\begin{array}{ll}
\left(\xi_{0}, 90^{\circ}\right) \Longrightarrow\left(\xi_{0}+\Delta \xi, 90^{\circ}\right), & \text { if }\left(0<U<P_{\xi_{+}}\right), \\
\left(\xi_{0}, 90^{\circ}\right) \Longrightarrow\left(\xi_{0}-\Delta \xi, 90^{\circ}\right), & \text { if }\left(P_{\xi_{+}}<U<P_{\max }\right), \\
\left(\xi_{0}, 90^{\circ}\right) \Longrightarrow\left(\xi_{0}, 90^{\circ}-\Delta \eta\right), & \text { if }\left(P_{\max }<U<1\right),
\end{array}
$$

where

$$
P_{\max }=P_{\xi_{+}}+P_{\xi_{-}} \cdot
$$

For the random walk computation along the line of symmetry $\left(\xi_{0}, 0^{\circ}\right)$, (8) becomes

$$
\begin{aligned}
V(i, 0)= & P_{\xi_{+}} V(i+1,0)+P_{\xi_{-}} V(i-1,0) \\
& +P_{\eta_{+}} V(i, 1) .
\end{aligned}
$$

The radial parts of the transition probabilities $\left(P_{\xi_{+}}\right.$and $\left.P_{\xi_{-}}\right)$ in (17) are the same as those in (14a) and (14b). The transition probability for the $\eta$ coordinate component of (17) is

$$
P_{\eta^{+}}=\left(\frac{\left[1 /(\Delta \eta)^{2}\right]}{\left[\left(1 /(\Delta \xi)^{2}\right)+\left(1 /(\Delta \eta)^{2}\right)\right]}\right) .
$$

Note also that $P_{\xi_{+}}+P_{\xi_{-}}+P_{\eta_{+}}=1$. For dispersed particles at point $\left(\xi_{0}, 0^{\circ}\right)$, they have probabilities $P_{\xi_{+}}, P_{\xi_{-}}$, and $P_{\eta_{+}}$of moving to points $\left(\xi+\Delta \xi, 0^{\circ}\right),\left(\xi-\Delta \xi, 0^{\circ}\right)$, and $(\xi, \Delta \eta)$, respectively. The particle walking pattern of the particles is as follows:

$$
\begin{aligned}
& \left(\xi_{0}, 0\right) \Longrightarrow\left(\xi_{0}+\Delta \xi, 0\right), \quad \text { if }\left(0<U<P_{\xi_{+}}\right), \\
& \left(\xi_{0}, 0\right) \Longrightarrow\left(\xi_{0}-\Delta \xi, 0\right), \quad \text { if }\left(P_{\xi_{+}}<U<P_{T 0}\right), \\
& \left(\xi_{0}, 0\right) \Longrightarrow\left(\xi_{0}, \Delta \theta\right), \quad \text { if }\left(P_{T 0}<U<1\right),
\end{aligned}
$$

where

$$
P_{T 0}=P_{\xi_{+}}+P_{\xi_{-}} .
$$

The potential at a specific point $\left(\xi_{0}, \eta_{0}\right)$ is to be determined. We define the transition probability $P_{n}$ as the probability that a random walk starting at the point of interest $\left(\xi_{0}, \eta_{0}\right)$ ends at the boundary node $\left(\xi_{n}, \eta_{n}\right)$ with prescribed potential $\left(V_{b}(n)\right)$.

Large particles are dispatched at the free node $\left(\xi_{0}, \eta_{0}\right)$. The application of Exodus method begins by setting particles $P\left(\xi_{i}, \eta_{j}\right)=0$ at all other nodes (both fixed and free), except at the free node $\left(\xi_{0}, \eta_{0}\right)$, where we assume a large value $N$. By scanning the mesh as in finite difference analysis (FDM), the particles are dispatched at each free node to its neighboring nodes according to the random walk transition probabilities described above. Detailed description of this process is given in [10]. If $N$ and $N_{n}$ designate the total number of particles dispersed and the number of particles that have reached the boundary $n$, respectively, the probability that a random walk terminates on the boundary is

$$
P_{n}=\frac{N_{n}}{N}
$$

If there are $M$ boundaries or fixed nodes (excluding the corner points), the potential at the specified node $\left(\xi_{0}, \eta_{0}\right)$ is

$$
V\left(\xi_{0}, \eta_{0}\right)=\sum_{n=1}^{M} P_{n} V_{b}(n)=\frac{\sum_{n=1}^{M} N_{n} V_{b}(n)}{N} .
$$

Since there are just two boundaries (the inner and outer oblate shell surfaces) in this potential problem, (22) simplifies to

$$
V\left(\xi_{0}, \eta_{0}\right)=\frac{\left(N_{1} V_{b}(1)+N_{2} V_{b}(2)\right)}{N},
$$


TABlE 1: Comparing the Exodus solution with finite difference and exact solution.

\begin{tabular}{ccccc}
\hline \multicolumn{2}{c}{ Potential point } & Exact potential $(\mathrm{V})$ & $\begin{array}{c}\text { Exodus method } \\
\text { potential }(\mathrm{V})\end{array}$ & $\begin{array}{c}\text { Finite difference (FDM) } \\
\text { potential }(\mathrm{V})\end{array}$ \\
\hline 1.1 & $\eta$ & 57.1513 & 57.1542 & 57.1536 \\
1.2 & $66^{\circ}$ & 63.7529 & 63.7577 & 63.7566 \\
1.3 & $69^{\circ}$ & 69.8269 & 69.8328 & 69.8313 \\
1.4 & $72^{\circ}$ & 75.3997 & 75.4060 & 75.4043 \\
1.5 & $75^{\circ}$ & 80.5005 & 80.5067 & 80.5050 \\
1.6 & $78^{\circ}$ & 85.1601 & 85.1656 & 85.1642 \\
1.7 & $81^{\circ}$ & 89.4096 & 89.4141 & 89.4129 \\
1.8 & $84^{\circ}$ & 93.2798 & 93.2830 & 93.2822 \\
1.9 & $87^{\circ}$ & 96.8004 & 96.8021 & 96.8017 \\
\hline
\end{tabular}

where $V_{b}(1)$ and $V_{b}(2)$ are the prescribed potentials at the inner and outer oblate spheroidal shells, respectively.

The equation for the exact solution of the potential computation in oblate spheroidal shells is [9]

$$
\begin{aligned}
V= & V_{b}(1)+\left(V_{b}(2)-V_{b}(1)\right) \\
& *\left(\frac{\left[\operatorname{gd}(\xi)-\operatorname{gd}\left(\xi_{1}\right)\right]}{\operatorname{gd}\left(\xi_{2}\right)-\operatorname{gd}\left(\xi_{1}\right)}\right),
\end{aligned}
$$

where $V_{b}(1)$ and $V_{b}(2)$ represent the nonzero potential (Dirichlet boundary conditions) at the inner and outer conducting oblate spheroidal shells, respectively, as shown in Figure 3. Also, $\operatorname{gd}(\cdot)$ denotes the Gudermannian function [6] and is represented as

$$
\operatorname{gd}(\xi)=\sin ^{-1}(\tanh (\xi))
$$

\section{Results}

Equations (10a)-(23) and (24)-(25) are used to implement the computation of potential distributions within two conducting oblate spheroidal shells numerically and analytically, respectively. The results obtained are as shown in Table 1. The results obtained from exact solution (analytical), the Exodus method, and the explicit finite difference solutions are compared in Table 1. Same step size was used for both the Exodus method and the FDM and this accounted for the closeness in the computed results obtained. The Exodus solution results were very close to the results obtained from exact solution because of the fact that though the Exodus method is a probabilistic method, its operation does not depend on random number generation which ultimately depends on the computation accuracy of the machine involved.

\section{Conclusion}

The use of the Exodus method to compute potential distribution inside two conducting oblate spheroidal shells maintained at two potentials has been implemented in this paper. The results obtained agreed with those obtained using finite difference (FDM) solution and the exact solution method.
The Exodus method employed in this work can be said to be almost as accurate as the exact method when compared to the fixed random walk Monte Carlo method because the results obtained were as accurate as the exact method.

\section{Conflict of Interests}

The authors declare that there is no conflict of interests regarding the publication of this paper.

\section{References}

[1] W. L. Gates, "Derivation of the equations of atmospheric motion in Oblate Spheroidal Coordinates," Journal of the Atmospheric Sciences, vol. 61, pp. 2478-2487, 2004.

[2] A. M. Al-Jumaily and F. M. Najim, "An approximation to the vibrations of oblate spheroidal shells," Journal of Sound and Vibration, vol. 204, no. 4, pp. 561-574, 1997.

[3] V. Punzo, S. Besio, S. Pittaluga, and A. Trequattrini, "Solution of Laplace equation on non axially symmetrical volumes," IEEE Transactions on Applied Superconductivity, vol. 16, no. 2, pp. 1815-1818, 2006.

[4] R. F. Tuttle and S. K. Loyalka, "Gravitational collision efficiency of nonspherical aerosols II: motion of an oblate spheroid in a viscous fluid," Nuclear Technology, vol. 69, no. 3, pp. 327-336, 1985.

[5] P. W. Casper and R. B. Bent, "The effect of the earth's oblate spheroid shape on the accuracy of a time-of-arrival lightning ground strike locating system," in Proceedings of the International Aerospace and Ground Conference on Lightning and Static Electricity, vol. 2, pp. 81.1-81.8, The National Aeronautics and Space Administration, The National Interagency Coordination Group (NICG), and Florida Institute, 1991.

[6] O. D. Momoh, M. N. O. Sadiku, and S. M. Musa, "A fixed random walk Monte Carlo computation of potential inside two conducting oblate spheroidal shells," in Proceedings of the IEEE Southeastcon, pp. 196-200, Nashville, Tenn, USA, March 2011.

[7] O. D. Momoh, M. N. O. Sadiku, and C. M. Akujuobi, "Solution of axisymmetric potential problem in spherical coordinates using Exodus method," in Proceedings of the PIERS Conference, pp. 1110-1114, Cambridge, Mass, USA, July 2010. 
[8] M. N. O. Sadiku, Numerical Techniques in Electromagnetics with MATLAB, CRC Press, Boca Raton, Fla, USA, 3rd edition, 2009.

[9] R. S. Alassar and H. M. Badr, "Analytical solution of oscillating inviscid flow over oblate spheroids with spheres and flat disks as special cases," Ocean Engineering, vol. 24, no. 3, pp. 217-225, 1997.

[10] O. D. Momoh, M. N. O. Sadiku, and C. M. Akujuobi, "Potential distribution computation in conducting prolate spheroidal shells using the exodus method," IEEE Transactions on Magnetics, vol. 47, no. 5, pp. 1426-1429, 2011. 

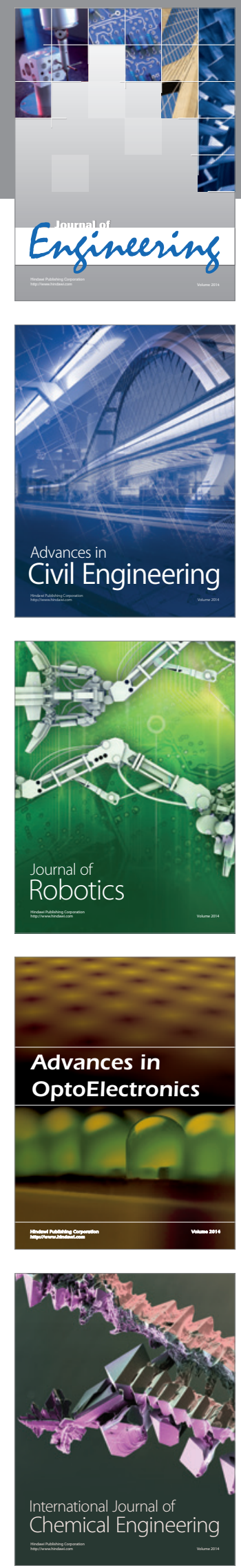

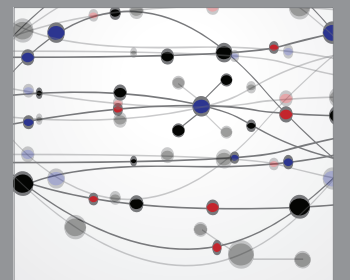

The Scientific World Journal
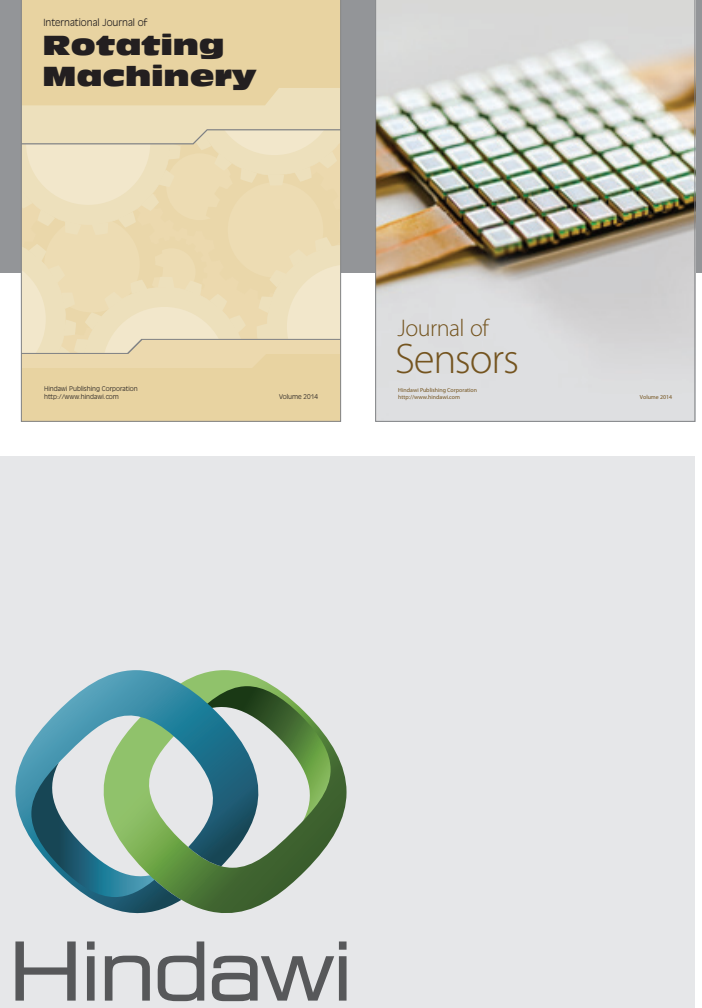

Submit your manuscripts at http://www.hindawi.com
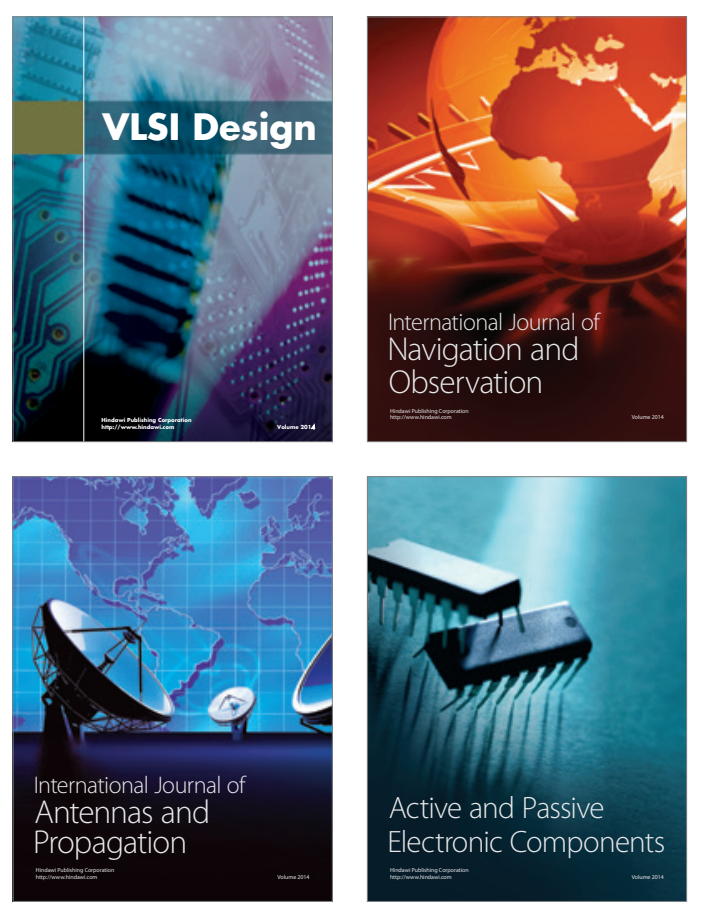
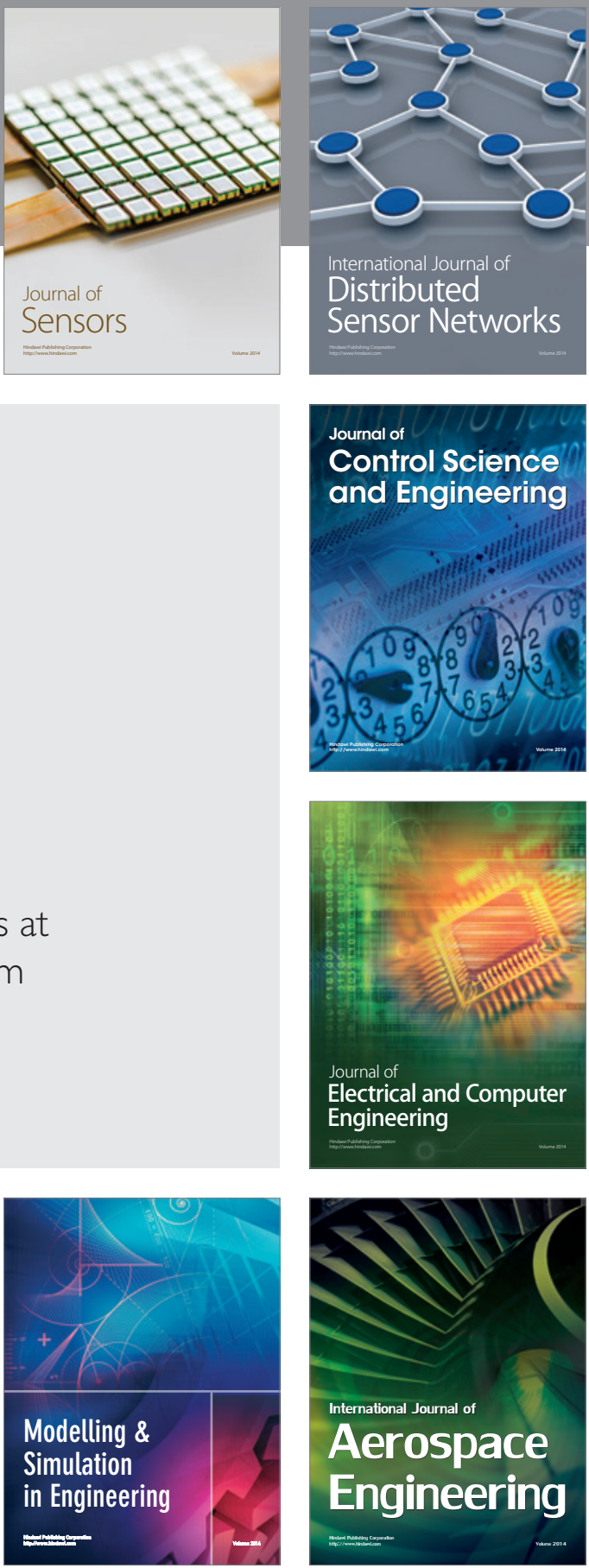

Journal of

Control Science

and Engineering
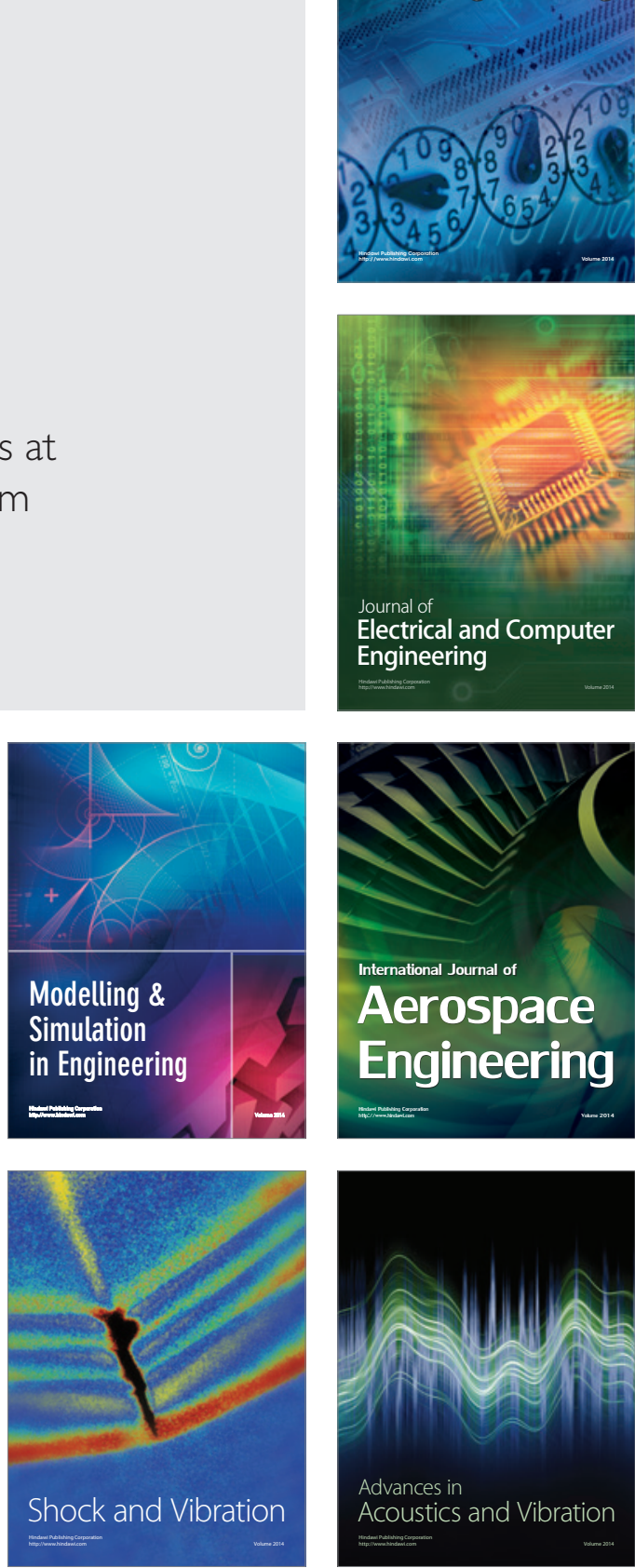